\title{
Synovial synthesis of rheumatoid factors and immune complex constituents in early arthritis
}

\author{
VALERIE JONES, P. C.R. TAYLOR, R. K. JACOBY, AND \\ T. B. W A L LINGTON \\ From the Postgraduate Medical School and Institute of Biometry and Community Medicine, University of \\ Exeter, and the South Western Regional Transfusion Centre, Bristol
}

SUMMARY Synovial synthesis of immune complex constituents and rheumatoid factors has been estimated in rheumatoid arthritis (RA) and in unclassifiable inflammatory arthritis (IA) and related to duration of symptoms. Within 6 months of onset of symptoms both RA and IA patients synthesised (or trapped) immune complexes locally. However, local synthesis of the rheumatoid factors was almost exclusive to RA patients and mainly in patients with symptoms of more than 6 months' duration. Thus all arthritis patients may respond immunologically to an initial trigger in the affected joint but only RA patients develop localised chronic autoimmunity later.

The optimum time to search for environmental factors which precipitate rhe umatoid arthritis should be during the earliest stages of the disease. ${ }^{1}$ Indeed immediate responses to environmental factors might be found in affected joints and precede the autoimmune response (rheumatoid factors) which is thought to precipitate the chronicity of rheumatoid disease. ${ }^{2}$

With a model of simple diffusion across the synovial membrane evidence has been obtained for synovial synthesis of immunoglobulins and rheumatoid factors. ${ }^{34}$ The concentration of a plasma protein in synovial fluid depends on the molecular size of that protein, on synovial membrane permeability which increases with inflammation, and on whether the protein is locally synthesised in the synovial membrane. With the use of plasma proteins whose site of synthesis is the liver, namely, orosomucoid, transferrin, ceruloplasmin and $\alpha_{2}$-macroglobulin, a linear relationship has been shown between log synovial fluid to serum concentration ratios and log molecular weight. ${ }^{3}$ When diffusion of immunoglobulins and IgM rheumatoid factor was calculated, concentrations were higher than predicted, indicating local synthesis of these components. ${ }^{34}$

We report here evidence, based on this diffusion model, of local synthesis of immune complex constituents and rheumatoid factors in 2 groups of patients, those with definite RA and those with unclassified inflammatory arthritis (IA). The results

Accepted for publication 15 March 1983.

Correspondence to $\mathrm{Dr}$ Valerie E. Jones, Postgraduate Medical School, Barrack Road, Exeter, Devon EX2 5DW. were then related to 3 stages of their disease, namely, less than 6 months, from 6 to 12 months, and after more than one year of arthritic symptoms. This extension of our earlier study ${ }^{5}$ suggests that immune complexes of unknown constitution are synthesised or trapped in inflamed joints in the early months, apparently before local synthesis of rheumatoid factors arises.

\section{Patients and methods}

Patients and samples. Eighty-seven patients were studied, 62 with definite RA (ARA criteria ${ }^{6}$ ) and 25 with unclassified inflammatory arthropathies; 36 of these patients were included in our earlier study. Two patients with noninflammatory arthritis were additional controls. Synovial fluid and paired blood samples were taken at the earliest feasible time in the disease, sometimes before definite RA was diagnosed. Many patients were taking nonsteroidal antiinflammatory drugs, and some had received intraarticular steroids some months previously. Among the RA patients none with symptoms of less than 6 months' duration had received second-line drugs. Of those with symptoms for more than 6 months 4 patients had taken low doses of steroids and 8 had been treated with penicillamine or gold for a short time. Four patients with longstanding RA had received second-line drugs in the past but had been without treatment for at least 6 months. Synovial fluids visibly contaminated with blood were discarded and fluids and sera were stored at $-20^{\circ} \mathrm{C}$. Serial samples from individual patients were not 
included, and when fluid was taken from 2 joints a mean value of the data was used.

Estimation of synovial synthesis. Three marker plasma proteins, albumin, ceruloplasmin, and $\alpha_{2}$ macroglobulin, were measured in serum (S) and synovial fluid (SF) by radial immunodiffusion. For 66 patients out of the 87 studied data were obtained for all 3 proteins. For the remaining 21 patients amounts of SF were limited, so only the albumin measurements were available. For each patient the ratios of their synovial fluid concentration to serum concentration (SF:S ratio) of the 3 marker proteins were used to estimate the corresponding ratios for immune complexes and rheumatoid factors. Rheumatoid factor (RF) was detected by latex agglutination (Latex-RF Reagent, Behringwerke AG, Germany). IgM RF and IgG RF were measured by radioimmunoassay, and concentrations are expressed as ng of ${ }^{125} \mathrm{I}$ anti- $\mu$ or anti- $\gamma$ bound per $10 \mu \mathrm{l}$ of sample ${ }^{7}$; levels above $49 \mathrm{ng}$ of anti- $\mu$ and $29 \mathrm{ng}$ of anti- $\gamma$ bound were above the normal serum range (mean +2 SD) for IgM RF and IgG RF respectively. Immune complexes (IC or $\mathrm{C} 1 \mathrm{q} \mathrm{BA}$ ) were measured by binding to fluid phase ${ }^{125} \mathrm{IC} \mathrm{C}$; binding activities of $20 \%$ or more were above the normal serum range. ${ }^{8}$ Calculations of synovial synthesis of IC, IgM RF, and IgG RF were derived from the following formulae:

(a) estimated concentration diffused from plasma $=$ estimated SF:S ratio $\times$ concentration in serum,

(b) synovial synthesis =

concentration in synovial fluid - estimated concentration diffused from plasma.

SF:S ratios were estimated from a linear relationship between the logarithm of the SF:S ratio and the logarithm of molecular weight as demonstrated by Kushner and Somerville ${ }^{3}$ :

$\log ($ SF:S ratio $)=$ patient 'factor' + ('slope' constant $\times \log$ (molecular weight))

where the patient 'factor' is the distinct intercept on the vertical axis for each patient, and the 'slope' constant is the same for each patient.

Fig. 1 gives a sample of the data plotted on logarithmic scales. The patient 'factors'-constant for each patient-and the 'slope' constant were estimated by means of least squares analysis on the logarithms of the SF:S ratios and the molecular weights of the 3 marker proteins.

\section{Results}

Clinical features. Patients were classified as RA if they had acquired 5 or more ARA criteria ${ }^{6}$ at the end of the study, whether or not a diagnosis could be made when samples were taken. All the IA patients

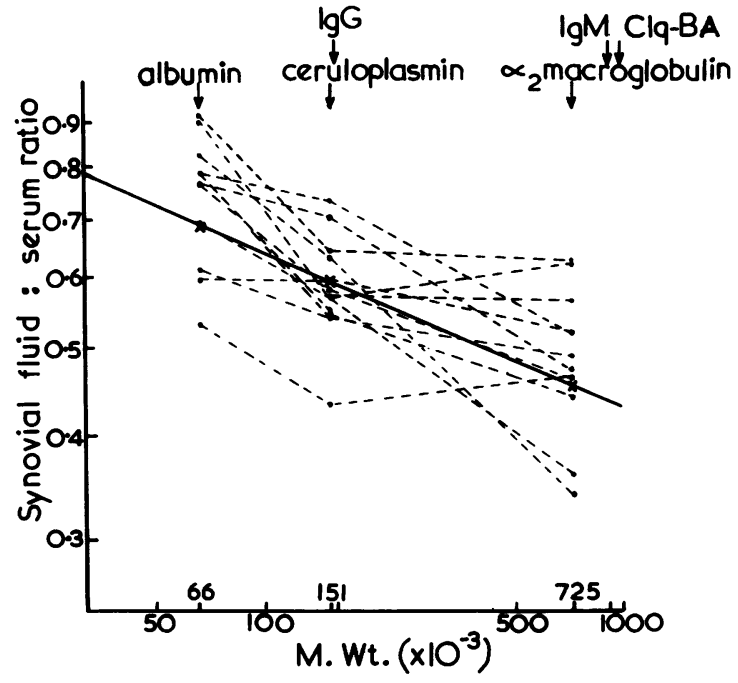

Fig. 1 Log SF:S ratios for albumin, ceruloplasmin and $\alpha_{2}$-macroglobulin plotted against their log molecular weights; 12 individual patients $\left(0_{-}-0_{-}\right)$and the regression line obtained with values from 66 patients $(\times-\times-\times)$.

had one or more of the first 5 ARA criteria and 2 IA patients were seropositive (criterion 8 ). Ten of the 25 IA patients had bilateral synovitis, but none had sufficient signs and symptoms for a diagnosis of definite RA. Patients were grouped according to duration of symptoms (Table 1) and not from the time of diagnosis of RA; those with symptoms for more than one year had suffered symptoms ranging from 13 months to 17 years.

Estimated diffusion from plasma. The concentrations of immune complexes, IgM RF and IgG RF in synovial fluid were plotted against the concentrations estimated to have diffused from plasma into synovial fluid across the synovial membrane (Fig. 2). Values on or near the $45^{\circ}$ dashed line indicate that the concentration in joint fluid was similar to the estimated

Table 1 Clinical data for rheumatoid arthritis $(R A)$ and inflammatory arthritis (IA) patients

\begin{tabular}{|c|c|c|c|}
\hline & & $R A$ & $I A$ \\
\hline \multicolumn{2}{|c|}{$\begin{array}{l}\text { Number of patients } \\
\text { Age (years); mean (range) } \\
\text { Males:females }\end{array}$} & $\begin{array}{l}62 \\
53(16-86) \\
16: 46\end{array}$ & $\begin{array}{l}25 \\
44(18-74) \\
10: 15\end{array}$ \\
\hline $\begin{array}{l}\text { No. of patients } \\
\text { with symptoms } \\
\text { for }\end{array}$ & $\begin{array}{l}<6 \text { months } \\
6-12 \text { months } \\
>12 \text { months }\end{array}$ & $\begin{array}{l}13 \\
22 \\
27\end{array}$ & $\begin{array}{r}11 \\
8 \\
6\end{array}$ \\
\hline $\begin{array}{l}\text { No. of seropositive } \\
\text { patients with } \\
\text { symptoms for }\end{array}$ & $\begin{array}{l}<6 \text { months } \\
6-12 \text { months } \\
>12 \text { months }\end{array}$ & $\begin{array}{r}6 \\
17 \\
21\end{array}$ & $\begin{array}{l}0 \\
2 \\
0\end{array}$ \\
\hline
\end{tabular}




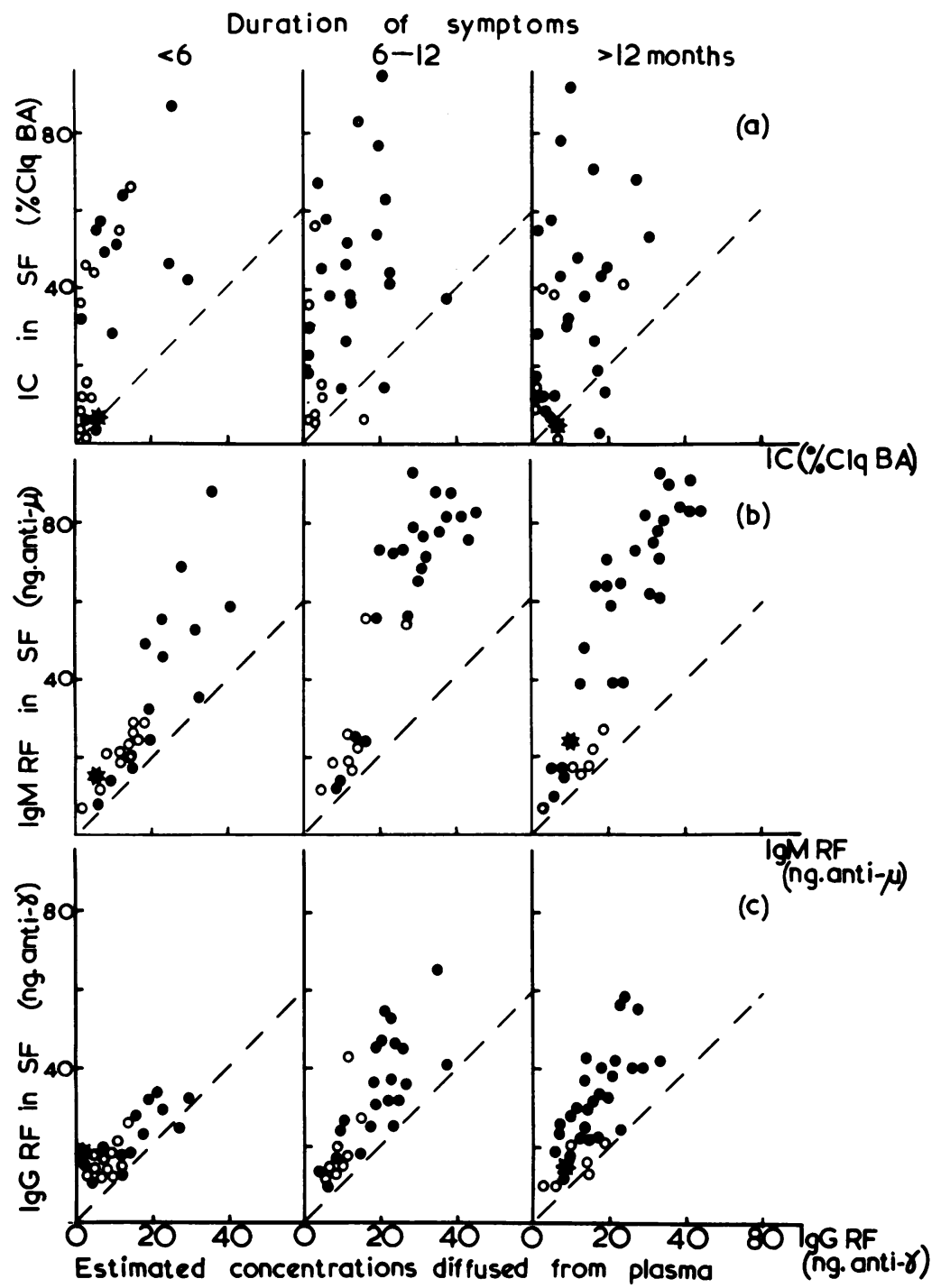

Fig. 2 Concentration in synovial fluid plotted against the estimated concentration derived from plasma of (a) immune complexes ( $\% \mathrm{Cl} q$ $B A$ ), (b) IgM RF (ng anti- $\mu$ bound/10 $\mu$ l), and (c) IgG RF (ng anti- $\gamma$ bound $/ 10 \mu l$ ) for each patient according to the duration of their symptoms. $=R A$ patients; $O=$ IA patients; $\star=$ noninflammatory arthritis.

amount derived from plasma. Values above the dashed line indicate a greater concentration in SF than diffused from plasma and thus suggust local synthesis of the appropriate constituent. Four patients, however, had values for immune complexes which lay below the line, implying that our model for simple diffusion across the synovial membrane may not be accurate for immune complexes. Nonetheless, the evidence indicates that IC are synthesised or trapped in the affected joints in the majority of both IA and RA patients. Furthermore, this synthesis or trapping takes place in the early months of symptoms as well as later in the disease, in contrast to rheumatoid factors. Evidence for local synthesis of both IgM and IgG RFs was detected almost exclusively in the RA patients (the exceptions were 2 IA patients with symptoms for 6 to 12 months) and mostly in RA patients having symptoms for 6 months or longer.

Estimates of local synthesis. The percentage of patients with their estimated concentrations of locally synthesised synovial fluid constituents are shown in Fig. 3. The 4 patients with small 'negative' estimates for immune complex synthesis were included with those having estimates of $1 \%$ to $10 \% \mathrm{C} 1 \mathrm{q}$ binding activity. Considerable amounts of immune complexes $(>20 \% \mathrm{C} 1 \mathrm{q}$ binding activity) were estimated to be formed locally by the majority of RA patients 


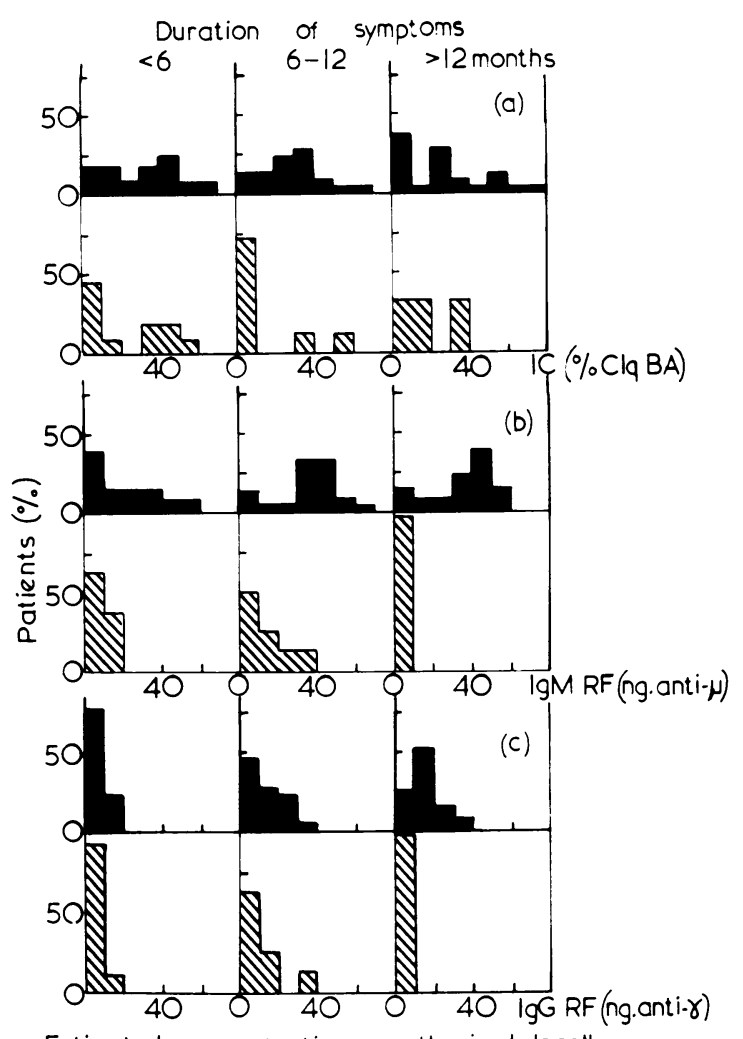

Estimated concentrations synthesised locally

Fig. 3 The percentage of $R A \square$ and $I A \mathbb{N}$ patients with estimated concentrations of (a) immune complexes (\% Clq $B A$ ), (b) IgM RF (ng anti- $\mu$ bound/10 $\mu$ l), and (c) IgG RF (ng anti- $\gamma$ bound $/ 10 \mu$ l) formed in their joints and according to the duration of their symptoms.

$(67 \%, 73 \%$, and $60 \%)$ and by about one-third of IA patients $(45 \%, 25 \%$, and $33 \%)$ at the first, second, and third stages of disease respectively. None of the IA patients (with 2 exceptions) synthesised large amounts of IgM RF locally ( $>20 \mathrm{ng}$ anti- $\mu$ bound). However, $46 \%$ of the RA patients were synthesising moderate amounts of IgM RF in the first months, and $82 \%$ and $78 \%$ were synthesising large amounts in the 2 later stages. A similar trend was seen for IgG RF in the RA patients; none were synthesising IgG RF in the early months ( $>20$ ng anti- $\gamma$ bound) and $27 \%$ and $22 \%$ were in the 2 later stages.

There was no significant correlation between concentrations of locally formed immune complexes (C1q BA) and locally synthesised rheumatoid factors (IgM RF) in RA or IA patients with symptoms for less than 6 months ( $p>0.3$ in both cases), suggesting that immune complexes appearing locally do not consist of rheumatoid factors at this early stage.

\section{Discussion}

These results suggest that immune complexes are formed or trapped in the inflamed joints of many arthritis patients in the early months of symptoms and that these IC are unrelated to rhe umatoid factors. Local synthesis of rhe umatoid factors appears to take place mainly in the later months and then almost exclusively in patients with definite RA. This confirms and extends our preliminary studies. ${ }^{5}$

The evidence is based on a model of simple diffusion across the synovial membrane, described by Kushner and Somerville. ${ }^{3}$ However, we have modified their statistical approach by introducing a patient 'factor' specific to each patient which represents that patient's average synovial permeability. This enabled us to obtain estimates of SF: $S$ ratios for immune complexes and rheumatoid factors specific to each patient rather than making global estimates for groups of patients. This model is appropriate to assess local synthesis of rheumatoid factors of both IgG and IgM class, ${ }^{4}$ because it has been convincingly shown that the synovial membrane offers no barrier to transport of rhe umatoid factors in either direction, from plasma to synovial space or vice versa. ${ }^{9}$

On the other hand the diffusion model may not be as suitable or accurate for immune complexes. First, the antigenic specificity of antigen-antibody complexes may differ in serum and synovial fluid; the specificity of the $\mathrm{C} 1 \mathrm{q}$ binding complexes detected in both compartments is unknown. Secondly, the estimates of IC diffused from plasma are based on a molecular size of $1 \times 10^{6}$ for these complexes and would be less accurate for larger or smaller complexes. If the complexes were larger, the amount diffused would be underestimated. In rheumatoid sera some complexes containing IgG have a molecular weight of $4 \times 10^{7}$, although the greatest proportion are approximately $2 \times 10^{6}$ (Svehag, personal communication). And $\mathrm{C} 1 \mathrm{q}$ binding complexes in synovial fluids are greater than $19 \mathrm{~S}$ from gel filtration experiments (our unpublished data). Nevertheless, it is worth noting that our estimate of the 'slope' constant in the log-linear relationship between SF:S ratio and molecular weight is $-0 \cdot 18$. This implies that a doubling of molecular weight leads to a reduction in SF:S ratio of only $12 \%\left(2^{-0 \cdot 18}=0 \cdot 88\right)$. In other words the estimation of concentration diffused from plasma is relatively insensitive to the errors introduced by inaccurate molecular weight determinations.

A third source of error might be the inability of large complexes to diffuse across the synovial membrane. Complexes of $1 \times 10^{6}$ should equilibrate freely in both directions, being only slightly larger than IgM, but larger complexes may not because there is evidence that aggregated $\lg G$ does not traverse the membrane from the synovial space direct 
to plasma but instead is removed via the lymphatics. ${ }^{10}$ Catabolism of large complexes is extremely rapid, however, both from the joint space and circulation, and therefore complexes should be equilibrated by catabolism as well as by diffusion. Despite all these reservations, our conclusions should be valid even if diffusion of immune complexes is overestimated or does not take place. If diffusion does not occur, the conclusion must be drawn that complexes in synovial fluid derive from locally synthesised constituents. In either event subtraction of estimated diffused components does introduce a more stringent assessment of local synthesis or trapping.

Our data show a rise in the proportion of RA patients who were locally synthesising rheumatoid factors as the duration of their symptoms increased. This suggests that local synthesis of the rheumatoid factors follows some time after the onset of symptoms and after detection of immune complexes. Several alternative but less likely explanations are possible. First, RA patients who have sizeable effusions in the first months of arthritis may be a selected group; for example, there is a low incidence of seropositivity $(45 \%)$ in this group of patients. Secondly, the radioassay may not detect rheumatoid factors with low affinity which might be formed in the early stages. Thirdly, Cecere et al. ${ }^{4}$ who have used a similar diffusion model, demonstrated local production of IgM RF, but unexpectedly their study provided no evidence for synovial synthesis of IgG RF. Indeed the converse was concluded, that in affected joints IgG RF was consumed by the rheumatoid inflammatory process. A direct comparison with our data cannot be made, because our samples are not treated with pep$\sin$ before the assay for IgG RF. Nonetheless, despite contrary conclusions concerning local synthesis of IgG RF, our results on IgM RF are not contested.

Evidence that $\mathrm{C} 1 \mathrm{q}$ binding immune complexes present in the early stages of disease are unrelated to rheumatoid factors is suggested by the lack of correlation between concentrations of the locally synthesised components. This is supported by previous studies in which circulating immune complexes were detected in the first months of symptoms in patients with unclassified arthritis and also in patients developing RA before their disease could be diagnosed as definite and before rheumatoid factor levels were raised. ${ }^{11}$ Thus we have further evidence that in RA patients immune complexes appear both in the circulation and in inflamed joints before evidence of autoimmunity appears. We are currently attempting to determine the composition of these complexes by physicochemical methods.

We thank Pamela Cowley, Elizabeth Cowdery, and Reg Czudek for expert technical assistance and Madeleine Parkin for secretarial help. This work was supported by a Project Grant from the Arthritis and Rheumatism Council to Dr V. E. Jones.

\section{References}

1 Schumacher H R. Synovial membrane and fluid morphologic alterations in early rheumatoid arthritis: microvascular injury and virus-like particles. Ann NY Acad Sci 1975; 256: 39-64.

2 Vaughan J H, Kaplan R A, Slovin S F, Carson D A. Biological properties of anti-IgG antibodies. In: Panayi G S, Johnson P M, eds. Immunopathogenesis of rheumatoid arthritis. Chertsey: Reedbooks, 1979: 56-9.

3 Kushner I, Somerville J A. Permeability of human synovial membrane to plasma proteins. Relationship to molecular size and inflammation. Arthritis Rheum 1971; 14: 560-70.

4 Cecere F, Lessard J, McDuffy S, Pope R M. Evidence for the local production and utilization of immune reactants in rheumatoid arthritis. Arthritis Rheum 1982; 25: 1307-15.

5 Jones V E, Jacoby R K, Cowley P J, Warren C. Immune complexes in early arthritis. II. Immune complex constituents are synthesised in the synovium before rheumatoid factors. Clin Exp Immunol 1982; 49: 31-40.

6 Ropes M W, Bennett G A, Cobb S. Diagnostic criteria for rheumatoid arthritis, 1958 revision. Ann Rheum Dis 1959; 18: 49-53.

7 Jones V E, Cowley P J, Allen C, Elson C J. The isolation of immune complexes containing IgM rheumatoid factor and recovery of IgG rheumatiod factor from the complexes. $J$ Immunol Methods 1980; 37: 1-14.

8 Verrier-Jones J, Cumming R H. Tests for circulating immune complexes. In: Thompson $\mathrm{R}$ A, ed. Techniques in clinical immunology. Oxford: Blackwell, 1977: 136-56.

9 Bluestone R, Cracchiolo A, Goldberg L S, Pearson C M. Catabolism and synovial transport of rheumatoid factor. Ann Rheum Dis 1970; 29: 47-55.

10 Sliwinski A J, Zvaifler $\mathrm{N} \mathrm{J}$. The removal of aggregated and nonaggregated autologous gammaglobulin from rheumatoid joints. Arthritis Rheum 1969; 12: 504-14.

11 Jones V E, Jacoby R K, Wallington T, Holt P. Immune complexes in early arthritis. I. Detection of immune complexes before rheumatoid arthritis is definite. Clin Exp Immunol 1981; 44: 512-21. 